\title{
Cyclophosphamide in steroid refractory unclassifiable idiopathic interstitial pneumonia and interstitial pneumonia with autoimmune features (IPAF)
}

To the Editor:

Unclassifiable idiopathic interstitial pneumonia (IIP) accounts for $\sim 15 \%$ of interstitial pneumonia diagnoses [1-3], and is therefore an important category in the daily practice of hospitals specialised in interstitial lung disease (ILDs). In unclassifiable IIP patients a confident diagnosis is not established despite a thorough diagnostic work-up and multidisciplinary discussion.

Management of unclassifiable IIP patients is based on disease behaviour and the most probable diagnosis, as proposed by international guidelines [2]. The first choice of drug is usually corticosteroid treatment, but not all patients respond to this treatment. Literature on treatment of progressive steroid refractory patients is scarce. Cyclophosphamide proved to be effective as a stabilising immunosuppressive agent in progressive ILD related to scleroderma [4,5]. At St Antonius Hospital, unclassifiable IIP patients refractory to corticosteroid treatment are empirically treated with intravenous cyclophosphamide pulse therapy (ICPT) with the objective to stabilise disease.

A subcategory of unclassifiable IIP consists of patients recently provisionally classified as "interstitial pneumonia with autoimmune features (IPAF)". IPAF patients do not meet the criteria for connective tissue disease (CTD), but do demonstrate clinical, serological or morphological features of CTD or similar disease behaviour [6]. Due to their immune-mediated features, steroid refractory IPAF patients are treated with immunosuppressive agents like ICPT $[7,8]$, although data on therapeutic outcome is lacking.

In this retrospective cohort study, we describe the results of 6 months ICPT in corticosteroid refractory unclassifiable IIP and IPAF patients.

Medical records of all consecutive ILD patients treated with ICPT $(n=108)$ visiting the outpatient clinic at St Antonius ILD Center of Excellence, Nieuwegein between 2011 and April 2016 were retrospectively analysed. All diagnoses were established after a multidisciplinary discussion conforming to international guidelines [2,9]. We excluded 70 patients due to known causes, e.g. hypersensitivity pneumonitis and ILD-CTD patients. In 38 patients no diagnosis could be established using international guidelines. These patients were categorised as unclassifiable IIP and included in the study. 19 out of the 38 patients retrospectively fulfilled the provisional IPAF criteria at the time of initiation of ICPT.

All patients were refractory to corticosteroids before initiation of ICPT. This regimen initially started at $0.5 \mathrm{mg} \cdot \mathrm{kg}^{-1} \cdot \mathrm{day}^{-1}$, with a maximum of $60 \mathrm{mg}$ corticosteroids and tapered monthly to $0.15 \mathrm{mg} \cdot \mathrm{kg}^{-1} \cdot \mathrm{day}^{-1}$ in 6 months. No azathioprine or mycophenolate mofetil (MMF) was used at the time of ICPT initiation. A 4-week schedule of ICPT with six cycles and a dose of $15 \mathrm{mg} \cdot \mathrm{kg}^{-1}$ bodyweight was used. To diminish side-effects, patients received $200 \mathrm{mg}$ of Mesna (sodium 2-mercaptoethane sulfonate).

Analyses of forced vital capacity (FVC) change were performed in patients treated with at least four cyclophosphamide cycles and with available FVC data at the start, 3-12 months before, and after 6 months and 12 months of therapy ( \pm 2 months). The Wilcoxon signed rank test was applied to compare FVC change before and after therapy. The Mann-Whitney test was used to detect differences between groups.

@ERSpublications

Analysis of FVC shows that steroid refractory unclassifiable idiopathic interstitial pneumonia patients can benefit from cyclophosphamide treatment http://ow.ly/e5D630iXt3p

Cite this article as: Wiertz IA, van Moorsel CHM, Vorselaars ADM, et al. Cyclophosphamide in steroid refractory unclassifiable idiopathic interstitial pneumonia and interstitial pneumonia with autoimmune features (IPAF). Eur Respir J 2018; 51: 1702519 [https://doi.org/10.1183/13993003.02519-2017]. 
Of 38 unclassifiable IIP patients, seven died during ICPT, including five severely impaired patients who used $>4 \mathrm{~L}$ of oxygen at the start of ICPT. Furthermore, four patients discontinued within 4 months of ICPT because of adverse events. Due to missing data at set time-points, four patients were not eligible for FVC analysis.

Our results focus on the 23 unclassifiable IIP patients that were eligible for FVC analysis. All patients demonstrated fibrosis on the high-resolution computed tomography scan. However, none of the patients had a radiological or histological pattern of a usual interstitial pneumonia (UIP). 13 patients fulfilled IPAF criteria. Provisional diagnosis of IPAF was mostly based on serological $(n=10)$ and morphological $(n=10)$ domains. The most frequent serological feature present in IPAF was a rheumatoid factor $>2 \times$ the upper limit of normal $(n=6)$. Seven out of the 10 non-IPAF unclassifiable IIP patients could not undergo a surgical lung biopsy (SLB) due to several comorbidities. Furthermore, three patients with a SLB had no classifiable diagnosis. In six IPAF patients a SLB was performed with five biopsies demonstrating evidence of organising pneumonia in addition to fibrotic changes.

At baseline, pulmonary function tests were significantly lower in the non-IPAF unclassifiable IIP patients than in the IPAF patients. Mean FVC was $59.5 \%$ predicted in non-IPAF unclassifiable IIP and $73.4 \%$ predicted in IPAF $(\mathrm{p}=0.041)$ and mean diffusing capacity of the lung for carbon monoxide was $30.0 \%$ predicted in non-IPAF unclassifiable IIP and $43.5 \%$ predicted in IPAF patients $(\mathrm{p}=0.003)$. Most patients (87\%) were treated with additional corticosteroids, generally dosed at below $20 \mathrm{mg}$ a day at ICPT initiation with a mean dose of $15 \mathrm{mg}$ a day. After 6 months the mean dose was tapered to $11.2 \mathrm{mg}$ a day. Other baseline characteristics including age, sex, smoking status, prednisone use and bronchoalveolar lavage lymphocyte percentage were not significantly different between the two groups.

Before initiation of ICPT the mean FVC decline for all patients was $-14.8 \%$ and after ICPT the mean increase was $+2.7 \%(\mathrm{n}=23 ; \mathrm{p}=0.002$; figure 1 , all unclassifiable IIP). Dividing the group into IPAF and non-IPAF unclassifiable IIP patients showed a clinically relevant and significant increase of FVC in IPAF patients at 6 months after the start of ICPT: FVC changed from $-12.3 \%$ before to $+9.4 \%$ after start of $\operatorname{ICPT}(\mathrm{n}=13 ; \mathrm{p}=0.002)$.

In non-IPAF unclassifiable IIP patients the effect of treatment was less clear and suggested at most a decrease in the rate of disease progression. A nonsignificant reduction of FVC decline was observed 6 months after the start of ICPT (FVC changed from $-18.2 \%$ before to $-5.9 \%$ after; $n=10 ; p=0.241$ ).

In 13 IPAF and seven non-IPAF unclassifiable IIP patients FVC data was present at 12 months. A prolonged beneficial improvement of FVC after ICPT was identified in all 20 unclassifiable IIP patients after 12 months. However, consolidation therapy consisted of various therapies such as corticosteroids, azathioprine, MMF and rituximab.

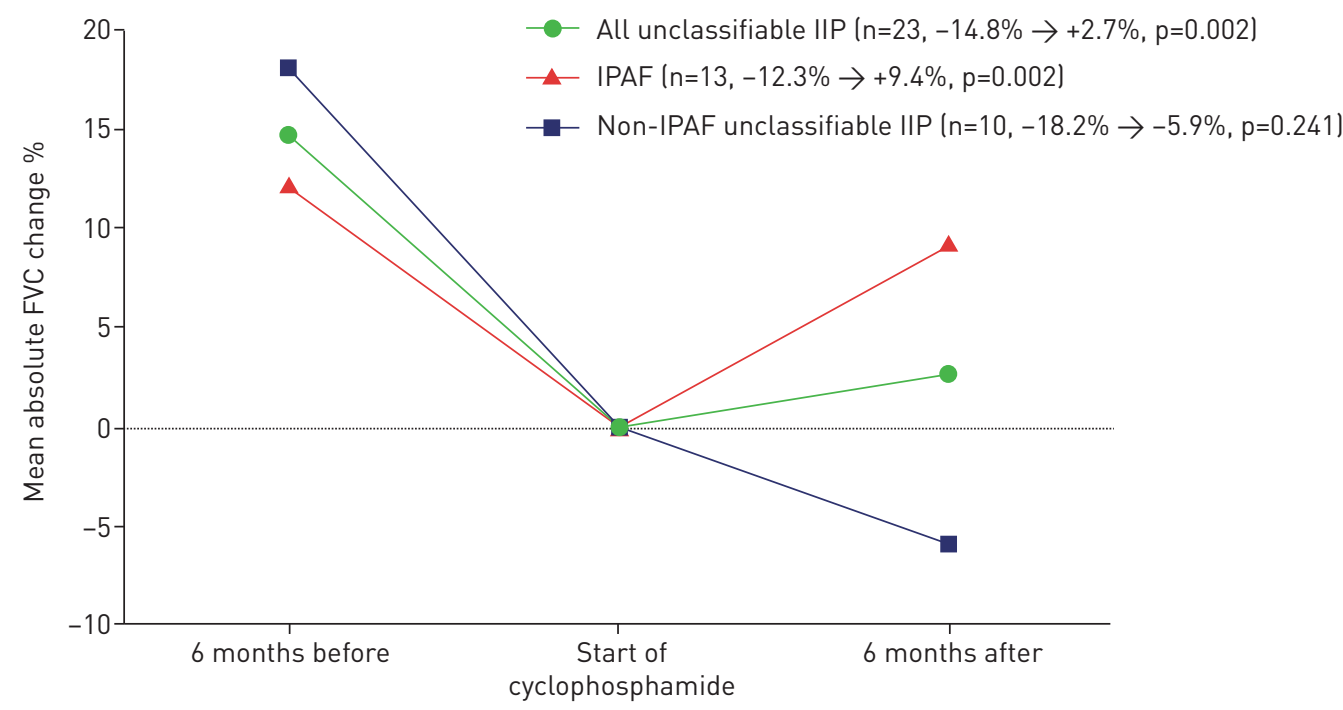

FIGURE 1 Mean percentage absolute forced vital capacity (FVC) change before and after initiation of intravenous cyclophosphamide pulse therapy. IIP: idiopathic interstitial pneumonia; IPAF: interstitial pneumonia with autoimmune features. 
No significant differences in the number of serious adverse events or missing data were observed between the two subgroups of IPAF and non-IPAF unclassifiable IIP patients.

To our knowledge, this is the first case series of unclassifiable IIP, including patients meeting IPAF criteria, describing the disease course with FVC data in a therapeutic setting. To date, therapeutic evidence was absent in this group of ILD.

Guidelines for unclassifiable IIP suggest that the therapeutic strategy should be based on disease behaviour [2]. Corticosteroids are generally given as the first line treatment in non-idiopathic pulmonary fibrosis (non-IPF) ILD patients. However, limited literature is available about therapeutic options in non-IPF ILD patients refractory to corticosteroids $[10,11]$. In IPF, immunosuppressive treatment proved to be harmful, while anti-fibrotic drugs were shown to slow down progressive disease. Recently, it was suggested that non-IPF ILD patients with progressive pulmonary fibrosis despite immunomodulatory agents might be treated with anti-fibrotic drugs [11]. However, we show that unclassifiable IIP patients might significantly benefit from ICPT and even recover part of their FVC loss.

Two published studies on IPAF characteristics reported that IPAF patients were mostly treated with steroids, azathioprine or MMF. However, no data on therapeutic outcome was provided. Both articles described a poor prognosis comparable with IPF $[7,8]$. In our study, disease progression of unclassifiable IIP patients fulfilling IPAF criteria prior to the start of ICPT is in line with these observations and a favourable response to ICPT was shown after 6 months. However, our cohort had no patients with a radiological UIP pattern $[12,13]$. Prolonged follow-up is needed to gain insight into the long-term effect of ICPT in this patient group.

Some of the limitations are the retrospective nature and relatively small size of our study. Selection bias of non-UIP and severely impaired, corticosteroid refractory patients from a tertiary centre must also be considered. Furthermore, we excluded patients with missing FVC data and patients that did not receive more than 4 months ICPT.

IPAF criteria were recently proposed to increase research on this particular group of ILD patients $[1,6]$. Our results suggest that use of these criteria also have potential value in therapeutic management of steroid refractory unclassifiable IIP patients $[1,14,15]$. However, prospective data are needed to validate our findings. Furthermore, studies with long term follow-up should explore tolerability and therapeutic outcome of azathioprine or MMF.

In conclusion, steroid refractory unclassifiable IIP patients treated with ICPT demonstrated a significant increase of FVC, especially those patients meeting the recently proposed IPAF criteria.

Ivo A. Wiertz ${ }^{1}$, Coline H.M. van Moorsel ${ }^{1,2}$, Adriane D.M. Vorselaars ${ }^{1}$, Marian J.R. Quanjel ${ }^{1}$ and Jan C. Grutters ${ }^{1,2}$ ${ }^{1}$ Interstitial Lung Diseases Center of Excellence, Dept of Pulmonology, St Antonius Hospital, Nieuwegein, The Netherlands. ${ }^{2}$ Division of Heart and Lungs, University Medical Centre Utrecht, The Netherlands.

Correspondence: Jan C. Grutters, Interstitial Lung Diseases Center of Excellence, Dept of Pulmonology, St Antonius Hospital, Koekoekslaan 1, 3435 CM Nieuwegein, The Netherlands. E-mail: j.grutters@antoniusziekenhuis.nl

Received: Dec 052017 | Accepted after revision: Feb 172018

Conflict of interest: I.A. Wiertz reports that this study is part of a research project (842002003) funded by ZonMw. He has received grants from Boehringer-Ingelheim (50\% of travel payment to attend the WASOG 2015 international congress), outside the submitted work. J.C. Grutters reports grants from Boehringer-Ingelheim (two advisory board fees of <1000 EUR; and four 50\% of travel payments to attend international congresses (ATS 2016, ATS 2015, WASOG 2015 and ATS 2014)), outside the submitted work.

Support statement: This study is part of a research project (842002003) funded by ZonMw. Funding information for this article has been deposited with the Crossref Funder Registry.

\section{References}

1 Ryerson CJ, Urbania TH, Richeldi L, et al. Prevalence and prognosis of unclassifiable interstitial lung disease. Eur Respir J 2013; 42: 750-757.

2 Travis WD, Costabel U, Hansell DM, et al. An official American Thoracic Society/European Respiratory Society statement: update of the international multidisciplinary classification of the idiopathic interstitial pneumonias. $A m$ J Respir Crit Care Med 2013; 188: 733-748.

3 Skolnik K, Ryerson CJ. Unclassifiable interstitial lung disease: a review. Respirology 2016; 21: 51-56.

4 Tashkin DP, Elashoff R, Clements PJ, et al. Cyclophosphamide versus placebo in scleroderma lung disease. $N$ Engl J Med 2006; 354: 2655-2666.

5 Hoyles RK, Ellis RW, Wellsbury J, et al. A multicenter, prospective, randomized, double-blind, placebo-controlled trial of corticosteroids and intravenous cyclophosphamide followed by oral azathioprine for the treatment of pulmonary fibrosis in scleroderma. Arthritis Rheum 2006; 54: 3962-3970. 
6 Fischer A, Antoniou KM, Brown KK, et al. An official European Respiratory Society/American Thoracic Society research statement: interstitial pneumonia with autoimmune features. Eur Respir J 2015; 46: 976-987.

7 Ahmad K, Barba T, Gamondes D, et al. Interstitial pneumonia with autoimmune features: clinical, radiologic, and histological characteristics and outcome in a series of 57 patients. Respir Med 2017; 123: 56-62.

8 Oldham JM, Adegunsoye A, Valenzi E, et al. Characterisation of patients with interstitial pneumonia with autoimmune features. Eur Respir J 2016; 47: 1767-1775.

9 Raghu G, Collard HR, Egan JJ, et al. An official ATS/ERS/JRS/ALAT statement: idiopathic pulmonary fibrosis: evidence-based guidelines for diagnosis and management. Am J Respir Crit Care Med 2011; 183: 788-824.

10 Schupp JC, Kohler T, Muller-Quernheim J. Usefulness of cyclophosphamide pulse therapy in interstitial lung diseases. Respiration 2016; 91: 296-301.

11 Kreuter M, Walscher J, Behr J. Antifibrotic drugs as treatment of nonidiopathic pulmonary fibrosis interstitial pneumonias: the time is now(?). Curr Opin Pulm Med 2017; 23: 418-425.

12 Collins B, Raghu G. Interstitial pneumonia with autoimmune features: the new consensus-based definition for this cohort of patients should be broadened. Eur Respir J 2016; 47: 1293-1295.

13 Fischer A, Collard HR, Cottin V, et al. Interstitial pneumonia with autoimmune features: the new consensus-based definition for this cohort of patients should be broadened. Eur Respir J 2016; 47: 1295-1296.

14 Troy L, Glaspole I, Goh N, et al. Prevalence and prognosis of unclassifiable interstitial lung disease. Eur Respir J 2014; 43: 1529-1530.

15 Cottin V, Wells A. Unclassified or unclassifiable interstitial lung disease: confusing or helpful disease category? Eur Respir J 2013; 42: 576-579. 